\title{
Hydrodynamic Signatures of Stationary Marangoni-Driven Surfactant Transport
}

\author{
M. M. Bandi, ${ }^{1, *}$ V. S. Akella, ${ }^{1}$ D. K. Singh, ${ }^{1}$ R. S. Singh, ${ }^{2}$ and S. Mandre ${ }^{2, \dagger}$ \\ ${ }^{1}$ Collective Interactions Unit, OIST Graduate University, Okinawa 904-0495, Japan \\ ${ }^{2}$ School of Engineering, Brown University, Providence, Rhode Island 02912, USA
}

(Received 2 May 2017; revised manuscript received 8 October 2017; published 28 December 2017)

\begin{abstract}
We experimentally study steady Marangoni-driven surfactant transport on the interface of a deep water layer. Using hydrodynamic measurements, and without using any knowledge of the surfactant physicochemical properties, we show that sodium dodecyl sulphate and Tergitol 15-S-9 introduced in low concentrations result in a flow driven by adsorbed surfactant. At higher surfactant concentration, the flow is dominated by the dissolved surfactant. Using camphoric acid, whose properties are a priori unknown, we demonstrate this method's efficacy by showing its spreading is adsorption dominated.
\end{abstract}

DOI: 10.1103/PhysRevLett.119.264501

Surfactants introduced at liquid interfaces give rise to Marangoni stresses that drive a flow [1]. The fundamental process of surfactant spreading, governed by its diffusion and transport via self-induced flow has many applications, from materials chemistry to biomechanics [2-10]. Many surfactants are soluble in the fluid and could be transported in a phase dissolved in the bulk or adsorbed at the interface $[11,12]$. A complete description of the resulting flow is hindered by the complexity of surfactant dynamics, which includes characterizing the equilibrium adsorption characteristics, the adsorption-desorption kinetics, and the transport by the flow $[13,14]$. Whereas methods based on molecular [15] or radiometric [16-18] markers can measure surface excess during a flow [19], low surfactant diffusivity into bulk fluid renders bulk concentration measurements at the interface difficult during flow. Direct Marangoni stress measurements via in situ surface tension gradient measurements are equally challenging. Simultaneous access to bulk and surface concentrations, Marangoni stress, sorption kinetics, and their subsequent correlation with one another to deduce the surfactant dynamics remains a formidable task.

In a recent study [11], for example, surfactant was introduced on the air-water interface through a steady point source. Simple scaling laws for surfactant spreading were derived by assuming the sorption kinetics to be much faster than the hydrodynamics, so that the dynamics were dominated by the dissolved phase. Verification of this assumption was not possible owing to the aforementioned difficulties. A possible alternative is that the sorption kinetics are too slow compared to the hydrodynamics, so that the dynamics are governed by the adsorbed phase. Either of these assumptions reduces the complexity of the

Published by the American Physical Society under the terms of the Creative Commons Attribution 4.0 International license. Further distribution of this work must maintain attribution to the author(s) and the published article's title, journal citation, and DOI. problem by enabling semianalytical steady solutions to the governing equations [20]. Our objective in this setting is an experimental validation of these assumptions using hydrodynamic measurements alone.

Consider a surfactant released steadily on the interface through a source much smaller in radial extent than the container size (see Fig. 1), such that a steady axisymmetric flow is established (see movie M1 in the Supplemental Material [21] for visualization). In a region much larger than the source but much smaller than the container, the source may be idealized as a point and the container assumed to be infinite. Furthermore, we consider the fluid viscosity and surfactant diffusivity to be small enough that most of the flow and surfactant concentration is established within a boundary layer near the surface. These approximations, along with the assumption of adsorption- or dissolution-dominated surfactant dynamics, render the governing physical description scale invariant. Consequently, the fluid radial $u(r, z)$ velocity components in cylindrical coordinates $(r, z)$ exhibit a self-similar structure [20]. Three experimentally measured invariant characteristics of this self-similar flow serve as hydrodynamic signatures of the simplified surfactant transport.

In this Letter, we present experimental verification of these flow signatures using two generic surfactants in

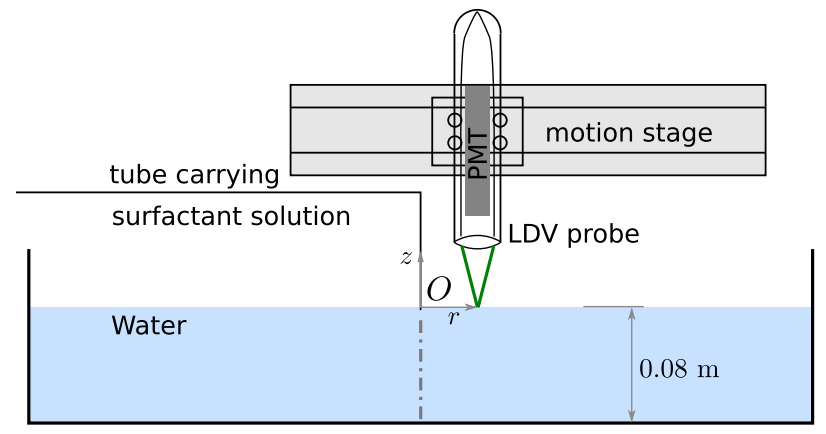

FIG. 1. Schematic of the experimental setup. 
water-sodium dodecyl sulphate (SDS) and Tergitol 15-S-9 (Tergitol). Both these surfactants are water soluble (solubility 0.2 and $0.7 \mathrm{~kg} / 1$, respectively) and span a range of critical micellar concentration (CMC) from $8 \times 10^{-5} \mathrm{mM}$ for Tergitol to $8 \times 10^{-3} \mathrm{mM}$ for SDS. SDS is ionic in nature, while Tergitol is nonionic. Without using any knowledge of the surfactant physicochemical parameters, we show that for concentrations less than $15 \%$ CMC, both surfactants exhibit flows dominated by adsorbed surfactant. In the same manner, mixture concentrations between $24 \%$ and 50\% CMC exhibit flow dominated by the dissolved surfactant. Finally, we also determine which of the two processes dominate the dynamics of a third surfactant, camphoric acid (CA), released at the interface from a gel tablet at unknown rates and concentrations.

A solution of SDS or Tergitol was introduced on an air-water interface via a borosilicate capillary (tip inner diameter of $3-5 \mu \mathrm{m}$ ) by Marangoni suction, a procedure empirically determined to minimize forcing a radial jet due to hydrodynamic pumping [22-24]. Four different concentrations for SDS and Tergitol ranging from about $0.05 \mathrm{CMC}$ to $0.5 \mathrm{CMC}$ (labeled $C 1-C 8$ in Fig. 2) were used to span the range of surfactant dynamics from adsorption dominated to dissolution dominated. CA was introduced on the interface through an agarose gel tablet (diameter $3 \mathrm{~mm}$, thickness $1 \mathrm{~mm}$ ) infused with CA (case $C 9$ in Fig. 2). The gel tablet was mounted on a vertical motion stage and brought in contact with the interface. In our experiments, the velocity boundary layer was minimally influenced by the dish bottom. The velocity profiles $u(r, 0)$ and $u\left(r=r_{1}, z\right)$, and the surface shear $u_{z}(r, z=0)$ of the axisymmetric flow that developed due to the Marangoni flow, were measured using laser doppler velocimetry (LDV).

The reproducibility required for the experiment and the measurement precision in velocity up to the fourth decimal place to ascertain the power laws and the boundary layer profile reported here require a tight protocol (for full experimental details, please see the Supplemental Material [21]).

Signature 1.-The measured surface radial velocity $u(r, 0)$ is shown on a logarithmic scale in Fig. 2(a). A correction to account for higher-order effects due to finite size of the CA tablet is applied, as detailed in the Supplemental Material. For all the nine cases considered, $u(r, 0)$ reaches a maximum $u_{\max }$ at $r=r_{\max }$ (about $1 \mathrm{~mm}$ ) and decays approximately as a power law in the range of radii $1<r / r_{\max }<20$. For $r / r_{\max } \gtrsim 20, u(r, 0)$ decreases much faster than the power-law decay.

The exponent of the power-law decay in the intermediate range of radii is the first hydrodynamic signature of the surfactant dynamics. In this range, five of the nine cases $(C 1, C 2, C 5, C 6$, and $C 9)$, those with surfactant concentrations $<0.15 \mathrm{CMC}$ and the one with $\mathrm{CA}$, exhibit an approximate decay of $u(r, 0)$ as $r^{-3 / 5}$. (For enhanced visibility, cool colors depict these cases in Figs. 2 and 5.) The remaining four cases $(C 3, C 4, C 7$, and $C 8)$,

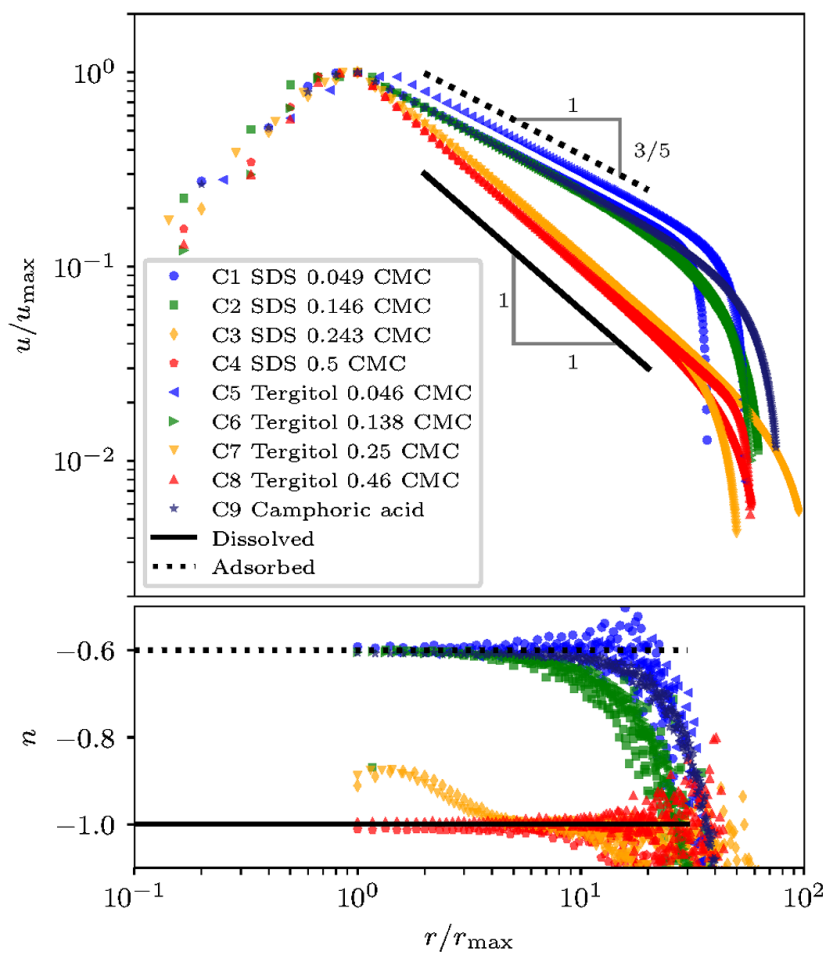

FIG. 2. (a) Radial velocity component $u(r, 0)$ at the fluid surface as a function of distance from the source center for three surfactants. Also plotted are power laws (1) and (2) expected for the dissolution-dominated (solid black line) and adsorptiondominated (dashed black line) cases. The velocity is rescaled by its maximum value $u_{\max }$ on the interface, and $r$ is rescaled by $r_{\max }$, the location where the maximum velocity occurs. (b) Same data as (a), but presented in the form of power-law exponent $n=d(\log u) / d(\log r)$.

which include surfactant concentration $>0.24 \mathrm{CMC}$ (shown in warm colors), exhibit decay as $r^{-1}$.

To confirm the measured slopes, Fig. 2(b) plots the log derivative (Selke's method [25]) $n=d \log u / d \log r$ as a function of $r$. The differentiation is performed using finite differences between neighboring experimentally measured data points. For the lowest concentrations of the SDS (0.049 CMC) and Tergitol (0.046 CMC), and in the range $1<r / r_{\max }<10$, the value of $n$ lies between -0.565 and -0.618 . For the next lowest concentration (0.146 CMC for SDS and $0.138 \mathrm{CMC}$ for Tergitol), $n$ departs from this range at $r / r_{\max } \gtrsim 8$. As the concentration is increased further (0.243 CMC for SDS and 0.25 CMC for Tergitol), $n$ lies in the range -0.87 to -1.04 , with a systematic departure from $\approx-1$ occurring in the range $1<r / r_{\max } \lesssim 4$. And finally, for the largest concentration (0.5 CMC for SDS and $0.46 \mathrm{CMC}$ for Tergitol), $n$ lies in the range -0.98 to -1.03 . For the flow driven by CA, $n$ lies in the range -0.60 to -0.63 . Based on these observations, we posit two values for the power-law exponents, $n \approx-0.6$ and $n \approx-1$, with the random variation attributed to measurement noise and the systematic deviations to departures from the asymptotic regimes of validity. 
These power laws can be understood in terms of the competing fluid and surfactant-induced stresses as follows. Due to self-similar nature of the flow, the length scale in the radial and depthwise directions are $r$ and the boundary layer thickness $\delta(r)$, respectively. Fluid inertia scales as $\rho u^{2} / r$ ( $\rho$ is fluid density), while viscous forces scale as $\mu u / \delta^{2}$ ( $\mu$ is dynamic viscosity). A balance between the two is expected in the boundary layer, which furnishes one relation, $\delta \sim \sqrt{\mu r / \rho u}$. Imposing the Marangoni stress, which scales as $\Delta \sigma / r(\Delta \sigma$ being the reduction in surface tension) to be equal to the scale of the fluid's shear stress, $\mu u / \delta$, leads to $\delta=\mu u r / \Delta \sigma$. The two cases are distinguished by the relation between $\Delta \sigma$ and the surfactant concentration, and how the surfactant is transported.

When the surfactant dynamics are dominated by the adsorbed phase, surfactant conservation implies $2 \pi u r c_{2}=q_{2}$, where $c_{2}$ is the surface concentration of the surfactant and $q_{2}$ is its surface flux. Here we neglect the diffusion of surfactant. The surface tension depends on surfactant concentration as $\Delta \sigma=-\Gamma_{2} c_{2}$, where $\Gamma_{2}$ is a proportionality constant. Eliminating $c_{2}$ and $\Delta \sigma$ leads to

$$
u(r, z=0)=f^{\prime}(0) C_{a} r^{-3 / 5}, \quad \delta_{a}(r)=r^{4 / 5} \sqrt{\nu / C_{a}},
$$

where $C_{a}=\left[\Gamma_{2}^{2} q_{2}^{2} \nu /\left(4 \pi^{2} \mu^{2}\right)\right]^{1 / 5}, f^{\prime}(0)$ is a dimensionless proportionality constant to be determined, and $\nu=\mu / \rho$.

When surfactant dynamics are dominated by the dissolved phase, surfactant bulk concentration $c_{3}(r, z)$ obeys an advection-diffusion equation with diffusivity $D$ and $\Delta \sigma=-\Gamma_{3} c_{3}$, where $\Gamma_{3}$ is a material-dependent constant. The surfactant diffuses in a boundary layer of thickness $\delta_{c}=\sqrt{D r / u}$, and hence surfactant conservation implies $2 \pi r u c_{3} \delta_{c} \propto q_{3}$, where $q_{3}$ is the volumetric surfactant release rate, which yields $c_{3} \propto q_{3} / \sqrt{u r^{3} D}$. The resulting Marangoni stress scales as $\Gamma_{3} c_{3} / r \propto \Gamma_{3} q_{3} / \sqrt{u r^{5} D}$, which balances the fluid viscous shear stress. The shear stress at the surface, due to a peculiarity in the boundary layer flow structure, does not scale as $\mu u / \delta$, but scales 1 order weaker in the small parameter $\delta / r$, as $\mu u / r$. Balancing the scales for Marangoni stress and shear stress yields

$$
u(r, z=0)=C_{d} r^{-1}, \quad \delta_{d}(r)=r \sqrt{\nu / C_{d}},
$$

where $C_{d}=\left[\Gamma_{3}^{2} q_{3}^{2} /\left(8 \pi^{3} \mu^{2} D\right)\right]^{1 / 3}$. These scaling estimates and the appropriate dimensionless proportionality constant are determined from an exact similarity solution by Bratukhin and Maurin [26] (for details, see Ref. [20]).

Signature 2.-To ensure that the power-law exponents arise due to the fluid dynamics presented here, and not due to any unexpected coincidences, we compare the depthwise profile $u\left(r_{1}, z\right)$ with theoretical expectations. In the case of adsorption-dominated surfactant dynamics, the solution may be expressed as

$$
u(r, z)=C_{a} r^{-3 / 5} f^{\prime}(\xi),
$$

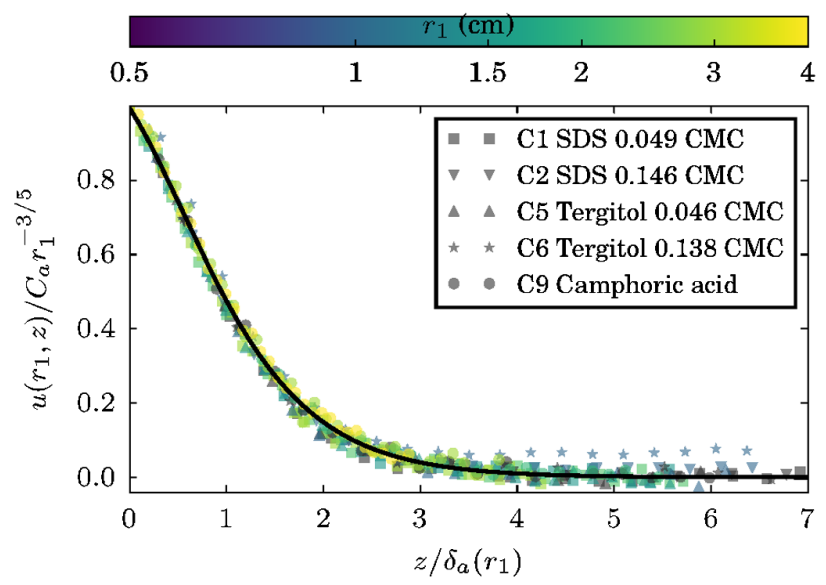

FIG. 3. The radial velocity profile in the boundary layer for flow dominated by adsorbed surfactant. The experimentally measured radial velocity profile (symbols) is normalized according to (3) and plotted against the similarity coordinate. Also plotted (solid curve) is the self-similar profile derived theoretically by solving (4).

in terms of a similarity coordinate $\xi=z / \delta_{a}(r)$ and a selfsimilar profile $f(\xi)$. Here $f$ satisfies [20]

$$
f^{\prime \prime \prime}(\xi)+\frac{3}{5} f^{\prime}(\xi)^{2}+\frac{6}{5} f(\xi) f^{\prime \prime}(\xi)=0,
$$

where $f(0)=0, f^{\prime \prime}(0) f^{\prime}(0)=\frac{2}{5}$, and $f^{\prime}(-\infty)=0$. This third-order ordinary differential equation is solved using a shooting method to obtain $f$, and the $u(r, z)$ is reconstructed using (3). The proportionality constant, $f^{\prime}(0) \approx$ 0.9943 in (1), is obtained as part of this solution.

Similarly, a leading-order approximation to the boundary layer flow profile driven by the surfactant whose dynamics are dominated by the dissolved phase [20] is

$$
u(r, z)=C_{d} r^{-1} \operatorname{sech}^{2}\left(\frac{z}{\delta_{d}(r) \sqrt{2}}\right)+O\left(\frac{\delta_{d}(r)}{r}\right) .
$$

Figures 3 and 4 show a comparison of experimentally measured depthwise profiles $u\left(r=r_{1}, z\right)$ for the cases exhibiting power-law exponents of $-3 / 5$ and -1 , respectively. The values of $C_{a}$ and $C_{d}$ are determined using the relations $u\left(r_{1}, 0\right)=C_{a} f^{\prime}(0) r_{1}^{-3 / 5}$ and $u\left(r_{1}, 0\right)=C_{d} / r_{1}$, which are subsequently used to determine the boundary layer thickness $\delta_{a, d}\left(r_{1}\right)$ for that profile. When the profiles are rescaled according to (3) or (5), and plotted against the similarity coordinate, they collapse close to universal curves. The theoretical profiles $f^{\prime}(\xi)$ and $\operatorname{sech}^{2}(\xi / \sqrt{2})$, respectively, well approximate these universal curves. Apart from random measurement noise, systematic departure of the data from these curves occurs due to two reasons: the return flow in the region outside the boundary layer, and departures from the power-law behavior at the measurement location $r=r_{1}$. This collapse validates the 


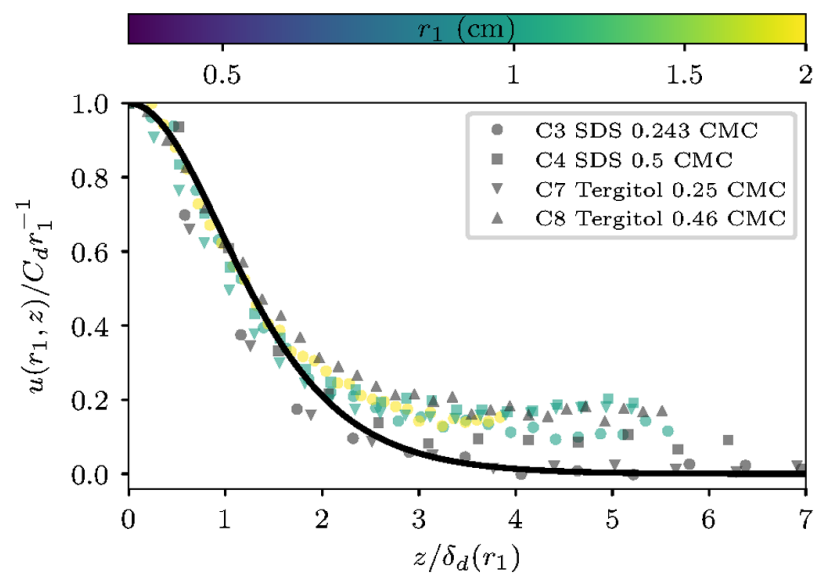

FIG. 4. The radial velocity profile in the boundary layer dominated by dissolved surfactant. The experimentally measured radial velocity profile (symbols), normalized by the scaling according to (5), is plotted against the similarity coordinate. Also plotted (solid curve) is the self-similar profile from (5).

thickness of the boundary layer arising from the adsorptionand dissolution-dominated regimes.

Signature 3.-The combination of radial decay as $r^{-3 / 5}$ and depthwise profiles shown in Fig. 3 is only possible when driven by an adsorbed layer of surfactant spreading as $2 \pi r u(r, 0) c_{2}(r)=q_{2}$, or a small perturbation thereof. However, the agreement in Fig. 4 of the measured velocity profile with the leading order of (5) is not conclusive proof of the flow being driven by a dissolved surfactant. It is so because, as explained in Ref. [20], Squire's radial jet [27] forced by a momentum source at the origin also exhibits $r^{-1}$ decay and the velocity profile (5) to leading order. Only higher-order corrections to the flow in the small parameter $\delta_{d} / r$ distinguish between Squire's radial jet and the complete solution (5). The shear rate $u_{z}(r, z=0)$ is such a quantity; $u_{z}=0$ for Squire's radial jet, and $u_{z}=2 u / r$ from the exact solution for dissolved surfactant-driven flow by Bratukhin and Maurin [26]. Based on this argument, we define the third hydrodynamic signature to be $\zeta=u_{z} l / u$ at $z=0$, where $l=\delta_{a}(r)$ if the surface velocity decays as $r^{-3 / 5}$, and $l=r$ if it decays as $r^{-1}$.

Figure 5 shows the experimentally measured values of $\zeta$ for all the nine cases. As expected, for cases $C 1$ and $C 5$ where adsorbed surfactant dominates the dynamics, $\zeta$ is scattered around the theoretically expected value $f^{\prime \prime}(0) / f^{\prime}(0) \approx 0.404$. For the cases $C 2$ and $C 6$, the reduction of $\zeta$ for $r \gtrsim 8 r_{\max }$ coincides with the departure of $n$ from $-3 / 5$. For the remaining cases, $\zeta$ is scattered around 2 and not around 0 , implying that the flow is driven by Bratukhin and Maurin's surfactant mechanism and not a localized momentum source near the origin.

Conclusion.-The agreement of the power-law exponent in Fig. 2, the depthwise profile in Figs. 3-4, and the dimensionless shear rate with the theoretically expected ones prove that the flow is driven by a surface stress caused by an agent transported in a manner homologous to the restrictive

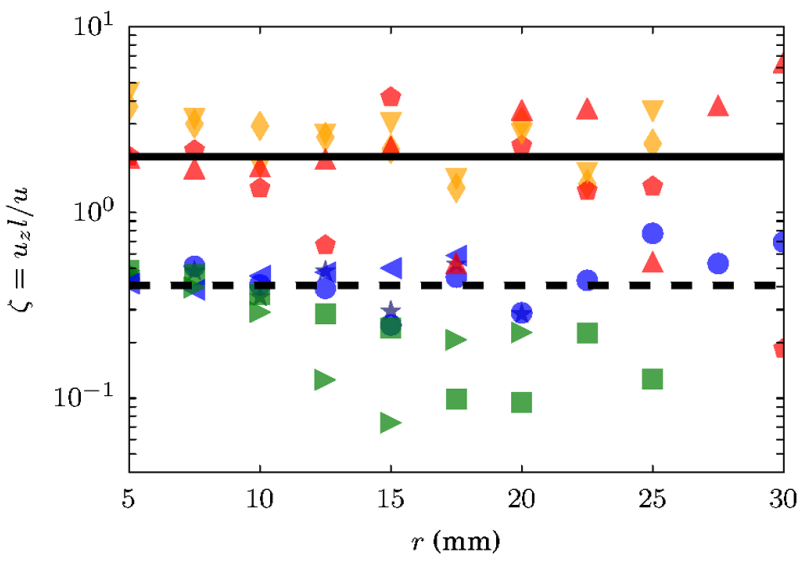

FIG. 5. Distribution of dimensionless shear stress on the interface. Legend is the same as Fig. 2.

assumptions underlying the theoretical derivation. Since our experimental protocol has carefully eliminated all other sources of surface stress, we are left with the unavoidable conclusion that the stress is caused by surfactant alone. Therefore, the surfactant dynamics within the power-law region in these cases must be as assumed in the theoretical model. In particular, for SDS and Tergitol released on the interface at concentrations $<0.14 \mathrm{CMC}$, the adsorbed surfactant governs the resulting dynamics, while for concentrations $>0.25 \mathrm{CMC}$, the dissolved surfactant dynamics dominates. A transition between the two behaviors is expected for intermediate concentrations, as suggested by the systematic deviations of $n$. For both surfactants, the deviation of $n$ from $-3 / 5$ towards -1 at $r / r_{\max } \gtrsim 8$ for cases $C 2$ and $C 6$ suggests the beginning of transition, and in the cases $C 3$ and $C 7$ at $r / r_{\max } \lesssim 4$ suggests the end of the transition. Given that the transition occurs within this range, it implies that the surfactant and hydrodynamic time scales approximately overlap, rendering simple order-ofmagnitude estimates unreliable to distinguish between the two regimes. Furthermore, there is no convenient independent way to measure a pivotal parameter in characterizing the dynamics - the fraction of the surfactant flux that is transported in an adsorbed phase. Therefore, using invariant hydrodynamic signatures to determine the validity of the assumptions about surfactant dynamics without a priori knowledge of the physicochemical parameters represents a fundamental advance on the topic.

Our result is quite robust, as we demonstrated for two surfactants varying in their CMC values by a factor of 100 , and can be used with other surfactants. We used these signatures to determine that CA released from a gel tablet spreads in an adsorbed phase, a result that bears upon Marangoni-driven self-assembly [28-33] and propulsion [34-37]. Assumptions about surfactant dynamics, such as those made in Ref. [11], can also be verified using the hydrodynamic signatures. A theoretical description of the transition between the two behaviors and its dependence on the physicochemical parameters remains to be developed. 
In closing, we note a vast majority of studies [38-45] to date have focused on transient Marangoni-driven surfactant spreading dynamics, where the flow ceases once the surfactant saturates the available interface area. Here, we have explored the much less studied class of statistically stationary Marangoni-driven flows [11,26,46,47] which arise when a mechanism for surfactant outflux balances its influx rate onto the interface, thus achieving a steady-state balance.

V. S. A., D. K. S., and M. M. B. were supported by the Collective Interactions Unit, OIST Graduate University. R.S.S. performed the research during internship with the Collective Interactions Unit, OIST Graduate University. The authors thank Kenneth J. Meacham III for technical support, Professor Amy Shen for help with tensiometry, Prof. Y. Yazaki-Sugiyama for help with pipette puller and Professor Walter Goldburg for the LDV equipment.

S. M. and M. M. B. conceived the study. M. M. B. designed and performed the experiments with assistance from R. S. S., D. K. S., and V.S.A., and also drafted the experimental protocol. The manuscript was drafted by S. M. in consultation with M. M. B. and was approved by all authors.

*Corresponding author.

bandi@oist.jp

${ }^{\dagger}$ Corresponding author.

shreyas_mandre@brown.edu

[1] V. G. Levich and V. S. Krylov, Annu. Rev. Fluid Mech. 1, 293 (1969).

[2] A. H. Jobe, N. Engl. J. Med. 328, 861 (1993).

[3] J. B. Grotberg, Annu. Rev. Fluid Mech. 26, 529 (1994).

[4] R. M. Harshey, Annu. Rev. Microbiol. 57, 249 (2003).

[5] T. Eisner, M. Eisner, and M. Siegler, Secret Weapons: Defenses of Insects, Spiders, Scorpions, and Other ManyLegged Creatures (Harvard University Press, Cambridge, MA, 2005).

[6] J. W. M. Bush and D. L. Hu, Annu. Rev. Fluid Mech. 38, 339 (2006).

[7] I. Cantat, S. Cohen-Addad, F. Elias, F. Graner, R. Höhler, O. Pitois, F. Rouyer, and A. Saint-Jalmes, Foams: Structure and Dynamics (Oxford University Press, New York, 2013).

[8] I. B. Ivanov and P. A. Kralchevsky, Colloids Surf. A 128, 155 (1997).

[9] G. T. Barnes, Agric. Water Manage. 95, 339 (2008).

[10] M. A. Herzig, G. T. Barnes, and I. R. Gentle, J. Colloid Interface Sci. 357, 239 (2011).

[11] M. Roché, Z. Li, I. M. Griffiths, S. Le Roux, I. Cantat, A. Saint-Jalmes, and H. A. Stone, Phys. Rev. Lett. 112, 208302 (2014).

[12] S. Le Roux, M. Roché, I. Cantat, and A. Saint-Jalmes, Phys. Rev. E 93, 013107 (2016).

[13] C.-H. Chang and E. I. Franses, Colloids Surf. A 100, 1 (1995).

[14] J. Eastoe and J. S. Dalton, Adv. Colloid Interface Sci. 85, 103 (2000).

[15] J.R. Lu, R. K. Thomas, and J. Penfold, Adv. Colloid Interface Sci. 84, 143 (2000).
[16] D. J. Salley, A. J. Weith, A. A. Argyle, and J. K. Dixon, Proc. R. Soc. A 203, 42 (1950).

[17] R. Matuura, H. Kimizuka, S. Miyamoto, and R. Shimozawa, Bull. Chem. Soc. Jpn. 31, 532 (1958).

[18] K. Tajima, M. Muramatsu, and T. Sasaki, Bull. Chem. Soc. Jpn. 43, 1991 (1970).

[19] S. Manning-Benson, S. R.W. Parker, C.D. Bain, and J. Penfold, Langmuir 14, 990 (1998).

[20] S. Mandre, J. Fluid Mech. 832, 777 (2017).

[21] See Supplemental Material at http://link.aps.org/ supplemental/10.1103/PhysRevLett.119.264501 for details of the experimental protocol and sample visualization.

[22] H. B. Squire, Q. J. Mech. Appl. Math. 4, 321 (1951).

[23] L. D. Landau and E. M. Lifshitz, Fluid Mechanics (Pergamon, New York, 1959).

[24] N. Laohakunakorn, B. Gollnick, F. Moreno-Herrero, D. G. A. L. Aarts, R. P. A. Dullens, S. Ghosal, and U. F. Keyser, Nano Lett. 13, 5141 (2013).

[25] W. Selke, Physica A (Amsterdam) 177A, 460 (1991).

[26] I. K. Bratukhin and L. N. Maurin, J. Appl. Math. Mech. 31, 605 (1967).

[27] H. B. Squire, in Fifty Years of Boundary Layer Research (Vieweg, Braunschweig, Germany, 1955), pp. 47-54.

[28] S. Soh, K. J. M. Bishop, and B. A. Grzybowski, J. Phys. Chem. B 112, 10848 (2008).

[29] N. J. Suematsu, S. Nakata, A. Awazu, and H. Nishimori, Phys. Rev. E 81, 056210 (2010).

[30] S. Soh, M. Branicki, and B. A. Grzybowski, J. Phys. Chem. Lett. 2, 770 (2011).

[31] E. Heisler, N. J. Suematsu, A. Awazu, and H. Nishimori, Phys. Rev. E 85, 055201 (2012).

[32] H. Kitahata, K. Iida, and M. Nagayama, Phys. Rev. E 87, 010901 (2013).

[33] B. A. Grzybowski, K. Fitzner, J. Paczesny, and S. Granick, Chem. Soc. Rev. 46, 5647 (2017).

[34] S. Nakata and M. Murakami, Langmuir 26, 2414 (2010).

[35] N. J. Suematsu, Y. Ikura, M. Nagayama, H. Kitahata, N. Kawagishi, M. Murakami, and S. Nakata, J. Phys. Chem. C 114, 9876 (2010).

[36] I. L. Liakos, P. Salvagnini, A. Scarpellini, R. Carzino, C. Beltran, E. Mele, V. Murino, and A. Athanassiou, Adv. Mater. Interfaces 3, 1500854 (2016).

[37] V. S. Akella, D. K. Singh, S. Mandre, and M. M. Bandi, arXiv:1701.06775.

[38] J. A. Fay, Oil on the Sea, edited by D. Hoult (Plenum, New York, 1969).

[39] D. W. Camp and J. C. Berg, J. Fluid Mech. 184, 445 (1987).

[40] O. Jensen and J. B. Grotberg, J. Fluid Mech. 240, 259 (1992).

[41] O. E. Jensen, J. Fluid Mech. 293, 349 (1995).

[42] A. D. Dussaud and S. M. Troian, Phys. Fluids 10, 23 (1998).

[43] A. D. Dussaud, S. M. Troian, and S. R. Harris, Phys. Fluids 10, 1588 (1998).

[44] D. W. Fallest, A. M. Lichtenberger, C. J. Fox, and K. E. Daniels, New J. Phys. 12, 073029 (2010).

[45] M. M. Bandi, T. Tallinen, and L. Mahadevan, Europhys. Lett. 96, 36008 (2011).

[46] Y. K. Bratukhin and L. N. Maurin, J. Eng. Phys. Thermophys. 14, 533 (1968).

[47] A. Zebib, G. M. Homsy, and E. Meiburg, Phys. Fluids 28, 3467 (1985). 